\title{
Investigation of magnetic flux gradients in hard superconductors
}

\author{
V. V. Bruk, V. V. Eremenko, N. I. Makedonskaya, Yu. A. Shabakayeva, \\ and V. A. Sirenko \\ B. Verkin Institute for Low Temperature Physics and Engineering of the National Academy of Sciences of Ukraine \\ 47 Lenin Ave., Kharkov 61164, Ukraine \\ E-mail: shabakayeva@ilt.kharkov.ua
}

Received October 26, 2000

\begin{abstract}
Analytical calculations of the transverse magnetostriction in a thin hard superconductor are presented in relation to the distributions of currents and fields within a superconducting specimen in a varied magnetic field. The approach is successfully tested on high-temperature superconductors. The flux distribution derived from magnetostriction measurements is in satisfactory agreement with that obtained from computer processing of magnetooptical images. The magnetic flux distribution below the irreversibility line of hard superconductors is derived from both original magnetization and magnetostriction measurements and image processing. Perfect consistency of the results is obtained for a family of high-temperature superconductors $\left(\mathrm{La}_{2-x} \mathrm{Sr}_{x} \mathrm{CuO}_{4}, \mathrm{Bi}_{2} \mathrm{Sr}_{2} \mathrm{CaCu}_{2} \mathrm{O}_{x}, \mathrm{YBa}_{2} \mathrm{Cu}_{3} \mathrm{O}_{7-\delta}\right)$.
\end{abstract}

PACS: $75.30 . \mathrm{Kz}$

\section{Introduction}

Measurements of the irreversible «giant» magnetostriction in HTSC [1-4] appears to be an effective tool for examination of the pinning-related phenomena in hard superconductors. The $1 D$ case was considered in detail for a thin long strip in a longitudinal magnetic field [5], and good agreement was achieved with the conventional models [6-9] of the magnetic flux distributions in type-II superconductors. In Ref. 10 another typical experimental situation is analyzed which corresponds to an external magnetic field normal to the flat face of a thin superconductor. Such a situation is frequently realized during magnetization and magnetic flux distribution measurements the thin films and single crystals of HTSC. It is not straightforwardly described by the classical models and their extensions, and it requires taking the demagnetizing effects into account. The problems of the current and flux distributions in these geometries are widely discussed in the literature (see, e.g., Ref. 11 and the references therein). In this work we present analytical estimations of the relevant magnetostrictions and show that their measurements can elucidate some aspects of the problem.

\section{Calculations}

It follows from Refs. 1-4 that the irreversible magnetostriction of HTSC with high critical current densities $j_{c}$ is caused by the pressure of the magnetic field, which smears the magnetic flux within a specimen with strong pinning. The relevant reduced strain of a superconductor in a magnetic field is related to the magnetic flux distribution inside the sample by the elastic equations taking into account the balance of forces in a system «flux line lattice imperfection», provided that the fully penetrated state has been established [1]:

$$
\frac{\Delta L}{L}=-\frac{1}{L} \int_{-L / 2}^{L / 2} \frac{B_{e}^{2}-B^{2}(x)}{\mu_{0} E} d x
$$

where $L$ is the sample size, $B_{e}=\mu_{0} H_{e}$ is the magnetic induction corresponding to the external magnetic field $H_{e}, B(x)$ is the local flux density in the sample, $E$ is the Young's modulus along the length change and $\mu_{0}$ is the permeability of vacuum. We consider a thin flat sample of thickness $d$ and width $2 a$ under the action of a varied field perpendicular to the face of the sample [10] (Fig. 1). If the specimen is assumed to be infinite in one direction $\mathbf{z}$ 


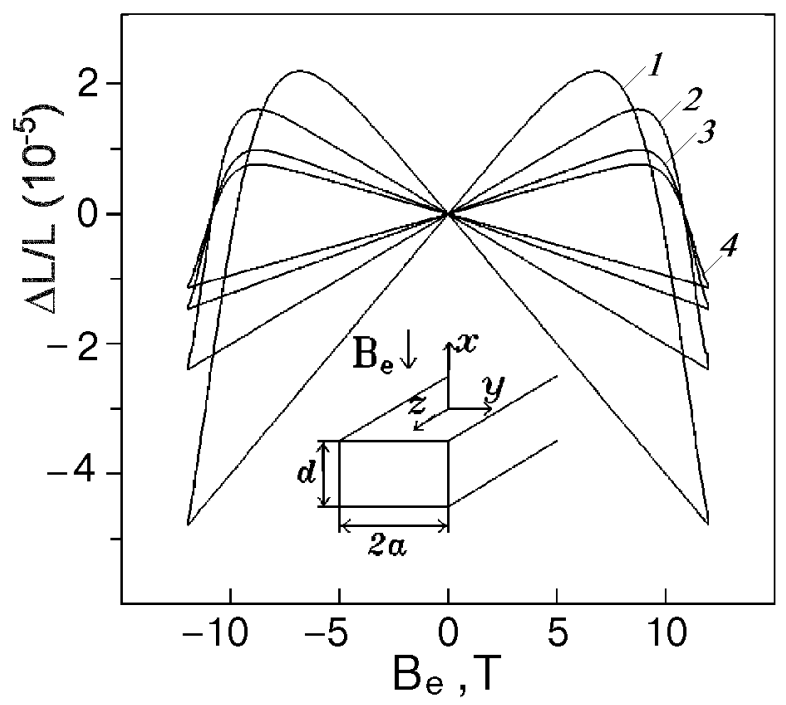

Fig. 1. Simulated magnetostriction loops of the $\mathrm{La}_{1.85} \mathrm{Sr}_{0.15} \mathrm{CuO}_{4}$ in a transverse magnetic field for different $h_{m}: 2.5(1) ; 5$ (2); 12.5 (3); 25 (4).

and $B_{e}$ to be uniform along the $\mathbf{x}$ axis, the flux gradients are in the $\mathbf{y}$ direction. In this case the longitudinal component of $B_{e}$ merely corresponds to the shielding currents, while the transverse component is responsible for the flux profiles, and so it alone determines the magnetostrictive strain, which is consequently directed along $y . B(y)$ is determined by the Maxwell equations.

The elastic stresses in the sample are calculated following [10]. Accordingly, the transverse magnetostriction (1) reads:

$$
\frac{\Delta L}{L}=\frac{1}{E} \int_{0}^{1} \sigma(\eta) d \eta
$$

which is equal to [10]

$$
\begin{gathered}
\frac{\Delta L}{L}=\frac{1}{E} \frac{\mu_{0} j_{c} d a}{\pi}\left[\frac{\pi}{2}\left(c_{m}-c_{1}\right) h_{m}+\frac{\pi c_{1}}{2} h-\right. \\
\left.-c_{m} \operatorname{Artanh}\left(\frac{\sqrt{k_{1}^{2}-k_{m}^{2}}}{k_{1}}\right)+c_{1}^{2} \operatorname{Artanh}\left(\frac{\sqrt{k_{1}^{2}-k_{m}^{2}}}{c_{m} k_{1}}\right)\right]+ \\
+\frac{1}{E} \frac{\mu_{0} j_{c} d a}{\pi} F\left(k_{m}, k_{1}\right)
\end{gathered}
$$

where $\sigma(\eta)$ is elastic stresses in the sample;

$$
\eta \equiv \frac{y}{a} \in[-1,1] ; c_{m} \equiv \sqrt{1-k_{m}^{2}}=\tanh \left(\pi h_{m}\right) \text {; }
$$

$$
\begin{gathered}
c_{1} \equiv \sqrt{1-k_{1}^{2}}=\tanh \left(\pi h_{1}\right) ; h_{m} \equiv \frac{B_{m}}{\mu_{0} j_{c} d} ; \\
h \equiv \frac{B_{e}}{\mu_{0} j_{c} d} ; h_{1} \equiv \frac{B_{m}-B_{e}}{2 \mu_{0} j_{c} d} ; \\
k_{1} \equiv \cosh ^{-1}\left(\pi h_{1}\right) ; k_{m} \equiv \cosh ^{-1} m\left(\pi h_{m}\right) ; \\
F\left(k_{m}, k_{1}\right)= \\
=\frac{2}{\pi}\left[\begin{array}{c}
k_{1} \\
k_{1} M\left(k_{m}, k_{1}, x=k_{1}\right)-\int_{k_{m}} M\left(k_{m}, k_{1}, x\right) d x
\end{array}\right] ;
\end{gathered}
$$

$B_{m}$ corresponds to the maximum field in a magnetization cycle.

The calculations of $\Delta L / L$ using flux gradients determined from magnetization measurements are presented in Fig. 1. It was anticipated that estimate of the flux gradients from magnetooptical image processing would also be used in these calculations. This is illustrated below on the $\mathrm{YBa}_{2} \mathrm{Cu}_{3} \mathrm{O}_{7-\delta}$ samples.

The process of determination of magnetic flux distribution from magnetooptical image consists of two stages using the magnetooptical method and the method of computer processing.

The principle of visualization of magnetic flux structure in high- $T_{c}$ superconductors, based on the Faraday effect, is shown in Fig. 2, $a$. The superconducting sample was covered with a garnet film doped with bismith. The external magnetic field was applied parallel to the $c$ axis of the sample. The magnetic flux profile was determined by observing changes of the domain structure. The domain structure (in a garnet film placed above the superconducting sample) was observed as a function of magnetic field intensity and may be regarded as magnetic flux sensor.

The visual pattern of magnetic flux trapping in the $\mathrm{YBa}_{2} \mathrm{Cu}_{3} \mathrm{O}_{7-\delta}$ sample when the external magnetic field is decreased from some maximum value to zero is shown in Fig. 2,b. The magnetooptical image obtained is subjected to preliminary computer processing.

Applying computer methods for image processing allowed us to examine the trapped magnetic flux distribution both on a qualitive level and to perform quantitative estimations of the local magnetic induction $\left(B_{e}\right)$ distribution over the sample surface. When a Bi-containing iron garnet film was used as 


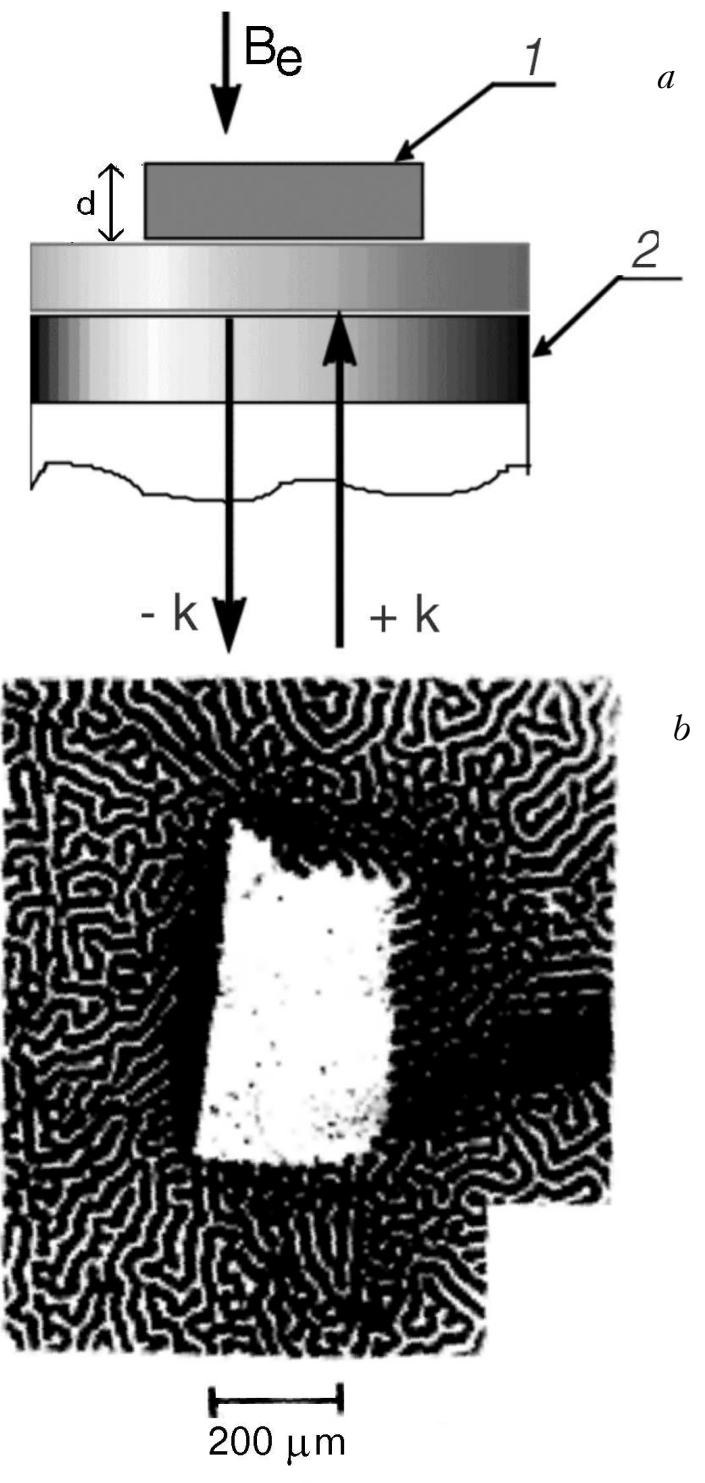

Fig. 2. Magnetooptical scheme: a) 1 - HTSC, 2 - indicator; $-\mathbf{k},+\mathbf{k}-$ transmitted and reflected light, respectively, $B_{e}-$ external magnetic field; $b$ ) visualization of trapping of magnetic flux in the sample of $\mathrm{YBa}_{2} \mathrm{Cu}_{3} \mathrm{O}_{7-\delta}(T=14 \mathrm{~K})$.

indicator, the preliminary stage of computer processing included low-frequency spatial image filtering in order to eliminate the disturbances induced by the labyrinth magnetic structure in the regions of $B_{e}$ below the saturation field. For this reason the initial image was scanned by a sliding window of square shape with a size a few times larger than the period of the labyrinth structure. For each selected window a two-dimensional Fourier transform was performed, the high-frequency harmonics associated with the labyrinth structure were filtered out, and inverse Fourier transform was performed. When a film with in-plane anisotropy was used as a indicator, there was no need of the preliminary image processing.
In order to obtain a quantitative estimate of the local magnetic induction after the low-frequency image filtering a segmentation of the latter was performed by separation of the regions with the comparatively uniform values of beam light intensity. This segmentation was based on analysis of a histogram of the numerical equivalents of the image brightness. In the interactive mode the brightness values were shown on the histogram, which corresponded to the boundaries between the separated grades of the image elements. To visualize the segmented image, all elements within the separated grade were coded by a definite color.

The transition to quantitative estimates was based on the following assumptions:

a) the angle between the transmission planes of the polarizer and analyzer did not differ much from the normal one;

b) trapped magnetic flux rotates the plane of polarization by small angle.

The first assumption means that the intensity of the registered light beam is linear in the second power of the sine of the rotation angle $\varphi$ of the plane of polarization: $I=I_{0} \sin ^{2} \varphi$. The second assumption allows the sine of the small angle to be replaced by the angle itself expressed in radians: $I \approx I_{0} \varphi^{2}$. Taking into account that $\varphi \sim B$, one obtains $I \sim \alpha^{2} B^{2}$, where $\alpha$ is a linear factor determined by the sample surface, $B$ is the integral value of the magnetic induction. A pattern of the obtained distribution of the local magnetic induction is shown in Fig. 3.

In addition the computer processing allows one to analyze the surface distribution of the local values of the $B_{e}$ and $B_{e}^{2}$ gradients, the latter being directly connected with the pinning force.

In order to study the spatial distribution of the $B_{e}^{2}$ gradient the image was spatially differentiated using the Sobel operator [12]. For this reason the projections of the image brightness gradients were calculated (in arbitrary integer units) along the axes $x$ and $y$ by the method of image convolution with masks of the Sobel operator:

$$
\begin{gathered}
H_{x}=\left[\begin{array}{lll}
-1, & 0, & 1 \\
-2, & 0, & 2 \\
-1, & 0, & 1
\end{array}\right] ; H_{y}=\left[\begin{array}{rrr}
1, & 2, & 1 \\
0, & 0, & 0 \\
-1, & -2, & -1
\end{array}\right], \\
\frac{\partial I}{\partial x(y)}\left(j_{x}, j_{y}\right)= \\
=\sum_{i_{x}=-1}^{1} \sum_{i_{y}=-1}^{1} I\left(j_{x}+i_{x}, j_{y}+i_{y}\right) H_{x(y)}\left(i_{x}+2, i_{y}+2\right),
\end{gathered}
$$




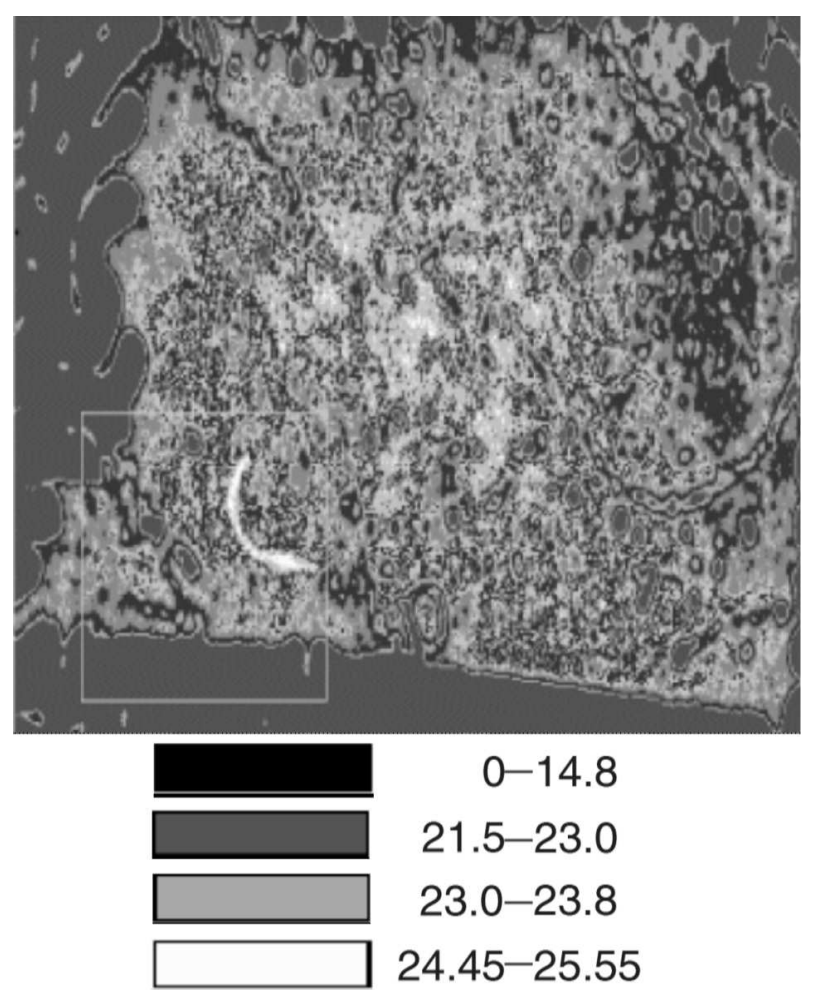

Fig. 3. Distribution of local magnetic induction, mT (filtered pattern).

where $j_{x}, j_{y}$ are the numbers of the row and column, respectively, with the intersection corresponding to the image element for which the gradient value is estimated.

Further, the modulus of the gradient is calculated according to the equation:

$$
\left|\frac{\partial B_{e}^{2}}{\partial \mathbf{r}}\right|=\frac{\alpha}{12 b}\left[\left(\frac{\partial I}{\partial x}\right)^{2}+\left(\frac{\partial I}{\partial y}\right)^{2}\right]^{1 / 2},
$$

where $b$ is the size of a single pixel of the image.

The appearance of the factor $\alpha / 12 b$ may be explained by the following equation:

$$
\begin{aligned}
& \frac{\partial B}{\partial x}=\frac{\alpha}{3}\left(\frac{I_{13}-I_{31}}{2 \sqrt{2} b} \frac{1}{\sqrt{2}}+\frac{I_{23}-I_{12}}{2 b}+\frac{I_{33}-I_{11}}{2 \sqrt{2 b}} \frac{1}{\sqrt{2}}\right)= \\
& =\frac{\alpha}{12 b}\left[2\left(I_{23}-I_{12}\right)+\left(I_{13}-I_{31}\right)+\left(I_{33}-I_{11}\right)\right],
\end{aligned}
$$

where $I_{i, j}$ is the intensity for the image element with the row number $i$ and column number $j$ in the selected window $(i, j=1-3)$. A similar formula may be written for $\partial B / \partial y$ also. Then

$$
\left|\frac{\partial B}{\partial \mathbf{r}}\right|=\left[\left(\frac{\partial B}{\partial x}\right)^{2}+\left(\frac{\partial B}{\partial y}\right)^{2}\right]^{1 / 2}
$$

In Fig. 4 a typical distribution of pinning force is presented. It is seen from the figure that the maximum values of $\left|\partial B_{e}^{2} / \partial \mathbf{r}\right|$ are observed within the boundary of the trapped magnetic flux region.

Estimaties of the $\left|\partial B_{e} / \partial \mathbf{r}\right|$ values were made in order to study the dependence of this characteristic on $B_{e}$ and hence to estimate magnetostriction with respect to (3).

The calculations were performed along different lines within the image, on going from the boundaries of the trapped magnetic flux region to the region of maximum values of $I$ using the following equations:

$$
\begin{gathered}
\left|\frac{\partial B_{e}}{\partial \mathbf{r}}\right|=\frac{1}{2 B_{e}}\left|\frac{\partial B_{e}^{2}}{\partial \mathbf{r}}\right|, \\
B_{e}=\alpha \sqrt{I} .
\end{gathered}
$$

For qualitative analysis of the obtained dependence the dispersion diagrams were constructed in the plane $B_{e}-\left|\partial B_{e} / \partial \mathbf{r}\right|$. A typical scatter diagram is presented in Fig. 5. As is seen from the figure the gradient initially increases with $B_{e}$ and then decreases.

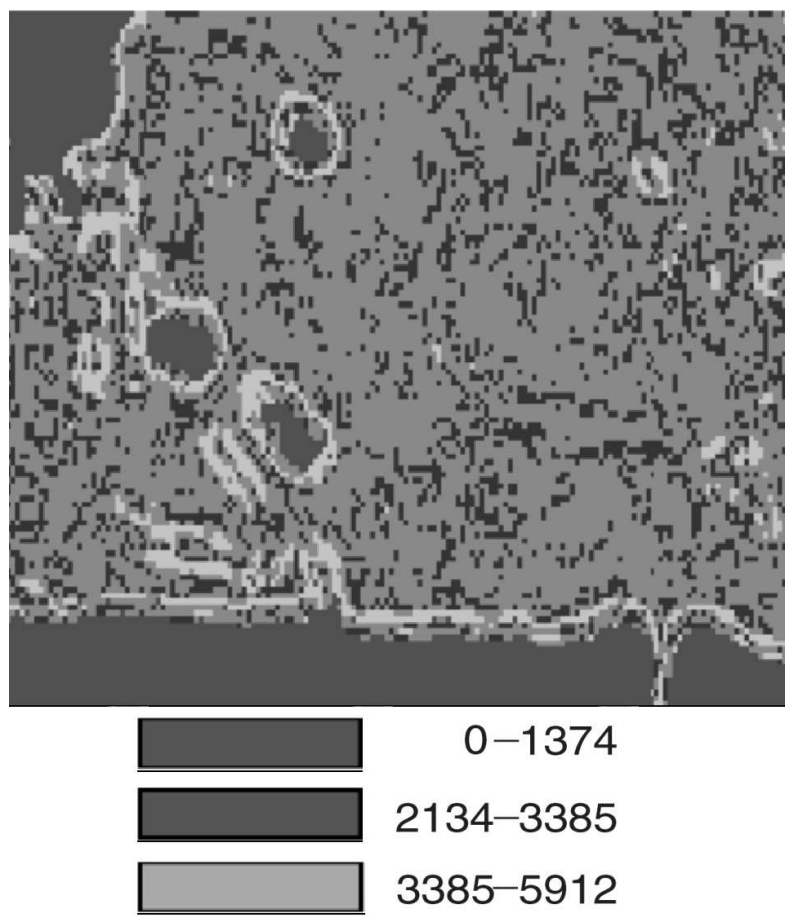

Fig. 4. Distribution of pinning force $F_{p}, \mathrm{~N} / \mathrm{mm}^{2}$ for a fragment outlined in Fig. 3. 


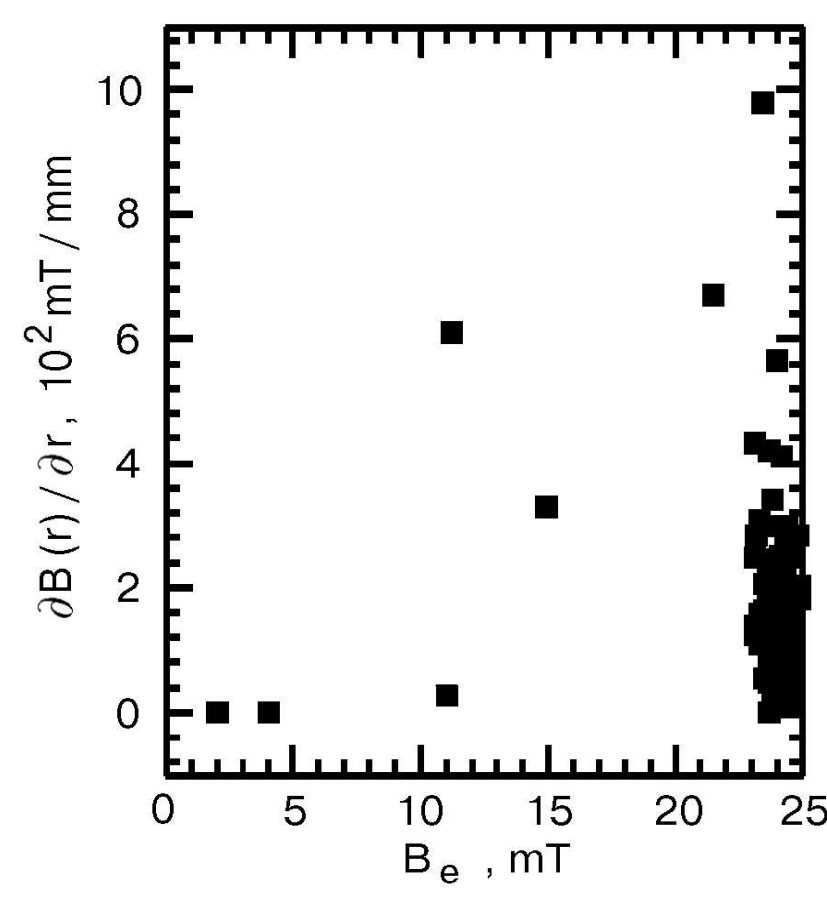

Fig. 5. Typical scattering diagram $B_{e}-\left|\partial B_{e} / \partial \mathbf{r}\right|$.

\section{Experimental data}

The magnetostriction and magnetization measurements were performed on a single crystal of $\mathrm{YBa}_{2} \mathrm{Cu}_{3} \mathrm{O}_{7-\delta}$. The superconducting transition temperature was determined from the temperature dependence of zero-field-cooled magnetization and equalled $T_{S N}=92 \mathrm{~K}$. The magnetostriction was measured by strain gauges along different crystallographic directions with an external magnetic field perpendicular to the face of the sample. The magnetization of the sample was measured in a vibrational magnetometer. The experimental geometry used allowed us to control the surface flux distributions with magnetooptical sensors by utilizing demagnetizing effects.
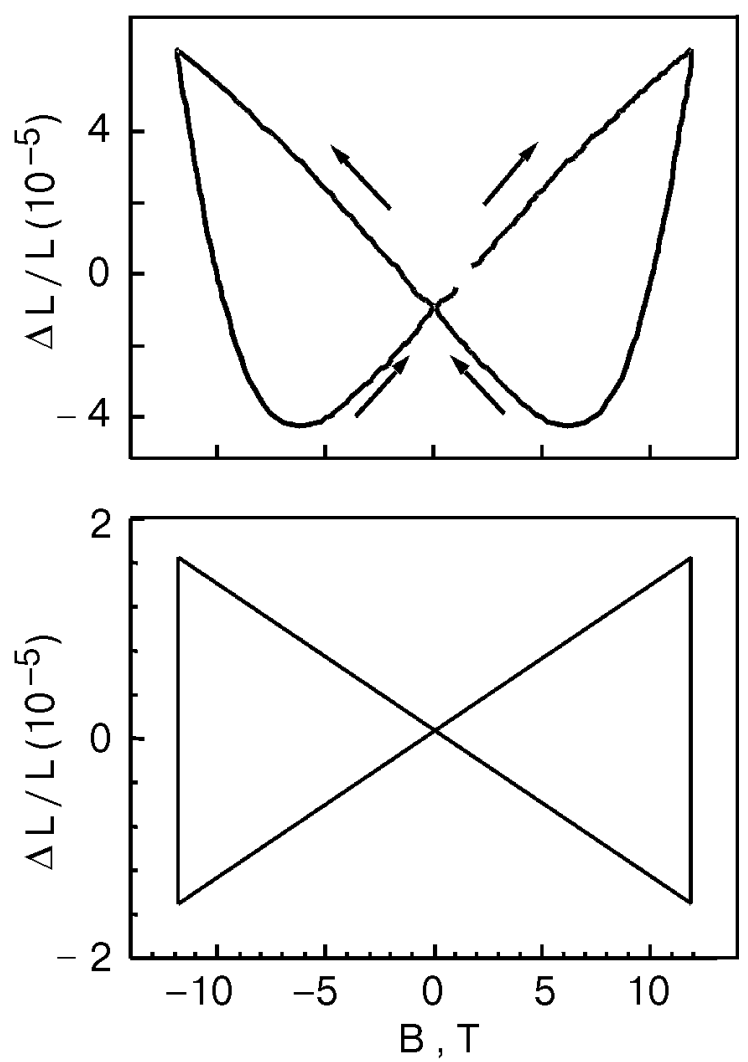

Fig. 6. Experimental and simulated magnetostriction loops for a $\mathrm{YBa}_{2} \mathrm{Cu}_{3} \mathrm{O}_{7-\delta}$ single crystal.

Figure 6 shows the magnetostriction of $\mathrm{YBa}_{2} \mathrm{Cu}_{3} \mathrm{O}_{7-\delta}$ at $14 \mathrm{~K}$. A more detailed analysis of the magnetostriction gives good agreement between the theory and experiment. We have obtained the width of the magnetostriction hysteresis loop at $14 \mathrm{~K}$ in a magnetic field of $12 \mathrm{~T}$ about $2 \cdot 10^{-5}$ both theoretically, using formula (3), and experimentally.

The Young's moduli of $\mathrm{YBa}_{2} \mathrm{Cu}_{3} \mathrm{O}_{7-\delta}$ along the direction of the magnetostriction measurements were calculated from the following equation:

$$
\begin{gathered}
\frac{1}{E}=\frac{c_{22} c_{33}-c_{23}^{2}}{c_{11} c_{22} c_{33}+2 c_{12} c_{13} c_{23}-c_{11} c_{23}^{2}-c_{22} c_{13}^{2}-c_{33} c_{12}^{2}}+ \\
+\frac{n_{y}^{2}\left[c_{33}\left(c_{11}-c_{22}\right)+c_{13}^{2}-c_{23}^{2}\right]+n_{z}^{2}\left[c_{22}\left(c_{11}-c_{33}\right)+c_{12}^{2}-c_{23}^{2}\right]}{c_{11} c_{22} c_{33}+2 c_{12} c_{13} c_{23}-c_{11} c_{23}^{2}-c_{22} c_{13}^{2}-c_{33} c_{12}^{2}}+ \\
+n_{x}^{2} n_{y}^{2}\left\{\frac{1}{c_{66}}+\frac{2\left(c_{13} c_{23}-c_{12} c_{33}\right)-\left(c_{22} c_{33}-c_{23}^{2}\right)-\left(c_{11} c_{33}-c_{13}^{2}\right)}{c_{11} c_{22} c_{33}+2 c_{12} c_{13} c_{23}-c_{11} c_{23}^{2}-c_{22} c_{13}^{2}-c_{33} c_{12}^{2}}\right\}+ \\
+n_{x}^{2} n_{z}^{2}\left\{\frac{1}{c_{44}}+\frac{2\left(c_{12} c_{23}-c_{22} c_{13}\right)-\left(c_{11} c_{22}-c_{12}^{2}\right)-\left(c_{22} c_{33}-c_{23}^{2}\right)}{c_{11} c_{22} c_{33}+2 c_{12} c_{13} c_{23}-c_{11} c_{23}^{2}-c_{22} c_{13}^{2}-c_{33} c_{12}^{2}}\right\}+
\end{gathered}
$$




$$
+n_{y}^{2} n_{z}^{2}\left\{\frac{1}{c_{55}}+\frac{2\left(c_{12} c_{13}-c_{11} c_{23}\right)-\left(c_{11} c_{33}-c_{13}^{2}\right)-\left(c_{11} c_{22}-c_{12}^{2}\right)}{c_{11} c_{22} c_{33}+2 c_{12} c_{13} c_{23}-c_{11} c_{23}^{2}-c_{22} c_{13}^{2}-c_{33} c_{12}^{2}}\right\}
$$

where the elastic moduli $c_{i k}$ are derived from the acoustic measurements [13], and $\mathbf{n}$ is the unit vector along a direction of magnetostriction measurement in the coordinates related to the principle lattice axes.

\section{Conclusion}

A model description of magnetostriction is proposed for a thin superconductor in a magnetic field perpendicular to a flat face of the sample. The simulated magnetostriction loop calculated using flux gradients derived from magnetooptical image processing is in a semi-quantitative agreement with the transverse magnetostriction measurements on the single crystal $\mathrm{YBa}_{2} \mathrm{Cu}_{3} \mathrm{O}_{7-\delta}$.

The authors acknowledge Dr. S. B. Feodosyev for his assistance in computations. We are grateful to Prof. H. Szymczak for stimulating discussions.
1. H. Ikuta, N. Hirota, Y. Nakayama, K. Kishio, and K. Kitazawa, Phys. Rev. Lett. 70, 2166 (1993).

2. C. de la Fuente, A. del Moral, J. I. Arnaudas, and J. S. Abell, Physica C244, 214 (1995).

3. L. Heil, H. Ikuta, K. Kishio, and K. Kitazawa, Physica C235-240, 2925 (1993).

4. A. Schmidt, F. Stellmach, and S. Ewert, Physica B194196, 1787 (1994).

5. H. Ikuta, K. Kishio, and K. Kitazawa, J. Appl. Phys. 76, 4776 (1994).

6. C. P. Bean, Phys. Rev. Lett. 8, 250 (1962); Rev. Mod. Phys. 36, 31 (1964).

7. Y. R. Kim, C. F. Hempstead, and A. R. Strnat, Phys. Rev. Lett. 9, 306 (1962); 12, 528 (1963).

8. V. R. Karasik, N. G. Vasil'ev, and V. G. Ershov, Zh. Eksp. Teor. Fiz. 59, 790 (1970) [Sov. Phys. JETP 32, 433 (1971)].

9. S. Senoussi, J. Phys. III (Paris) 2, 1041 (1992).

10. V. V. Eremenko, V. A. Sirenko, H. Szymczak, and A. Nabialek, Superlattices and Microstructures 24, 221 (1998).

11. E. H. Brandt, Phys. Rev. B54, 4246 (1996).

12. W. K. Pratt, Digital Image Processing, New York, WileyInterscience (1978).

13. H. Ledbetter and M. Lei, J. Mater. Res. 6, 2253 (1991). 